Article

\title{
Deep Learning of High-Resolution Aerial Imagery for Coastal Marsh Change Detection: A Comparative Study
}

\author{
Grayson R. Morgan ${ }^{1}$, Cuizhen Wang ${ }^{1}$, Zhenlong $\mathrm{Li}^{1 *}$, Steven R. Schill ${ }^{2}$ and Daniel R. Morgan ${ }^{3}$ \\ 1 Department of Geography, University of South Carolina, Columbia, SC, USA \\ 2 The Nature Conservancy, Caribbean Division, Coral Gables, FL 33134, USA \\ 3 Beaufort County Mapping and Applications Department, SC, USA \\ * Correspondence: zhenlong@sc.edu
}

\begin{abstract}
Deep learning techniques are increasingly being recognized as effective image classifiers. Aside from their successful performance in past studies, the accuracies have varied in complex environments in comparison with the popularly applied machine learning classifiers. This study seeks to explore the feasibility for using a U-Net deep learning architecture to classify bi-temporal high resolution county scale aerial images to determine the spatial extent and changes of land cover classes that directly or indirectly impact tidal marsh. The image set used in the analysis is a collection of a 1-m resolution collection of National Agriculture Imagery Program (NAIP) tiles from 2009 and 2019 covering Beaufort County, South Carolina. The U-net CNN classification results were compared with two machine learning classifiers, the Random Trees (RT) and the Support Vector Machine (SVM). The results revealed a significant accuracy advantage in using the U-Net classifier $(92.4 \%)$ as opposed to the SVM $(81.6 \%)$ and RT $(75.7 \%)$ classifiers for overall accuracy. From the perspective of a GIS analyst or coastal manager, the U-Net classifier is now an easily accessible nad powerful tool for mapping large areas. Change detection analysis indicated little areal change on marsh extent, though increased land development throughout the county has the potential to negatively impact the health of the marshes. Future work should explore applying the constructed UNet classifier to coastal environments in large geographic areas, while also implementing other data sources (e.g., LIDAR, multispectral data) to enhance classification accuracy.
\end{abstract}

Keywords: Deep Learning; Machine Learning; Change Detection; Coastal; Marsh; Remote Sensing; Aerial Imagery

\section{Introduction}

Machine learning (ML) algorithms have become commonplace in remote sensing data analysis [1-8]. The successful use of ML for a variety of GIS and remote sensing applications has led to the implementation of these methods, often based on support vector machine (SVM) and random forests (RF) statistical methods, into GIScience software packages that can be used by non-technical investigators. The tools are readily available for supervised classifications in particular [9-10]. Numerous studies have supported the use of machine learning over traditional, statistically-based classifiers such as Maximum likelihood methods, with SVM often performing the best [11-14]. ML classifiers have now been established across the professional community as reliable tools for mapping without requirement of extensive machine learning and programming experiences.

Advancements within the past ten years have led to a new division within machine learning. Deep Learning (DL) is a learning algorithm designed to mimic the function of human brain in the form of neural networks [15]. An advanced subsection of ML, DL is able to perform artificial intelligence functions with extensive training resources. The recent popularity and success of DL in other disciplines and applications such as speech recognition [16] and medical image recognition [17] has led to the rise of its use in remote sensing applications. While citing other reviews of DL applications in remote sensing by 
[18] and [19], [20] gave a comprehensive review by describing different model types of DL in remote sensing. The authors also found in their meta-analysis of the subject that, as of the publication of their article, there were 221 peer reviewed articles and 181 conference papers or proceedings pertaining to remote sensing and DL. It is clear that use-cases of DL in remote sensing applications are increasing rapidly.

As applications of DL classifiers in remote sensing become more established, the algorithms and tools using DL for image classification are being made available as userfriendly graphical user interface (GUI) tools in commonly used GIS software, just like the ML tools. Though geospatial software companies tout both user-friendly and robust DL tools, it is often difficult to manipulate the tools to the user's desired specifications. Nevertheless, just as the ubiquity of ML GUI tools opened the use of ML to users without a background in programming and ML, DL tools are also now available to all. Applicationdriven researchers, managers, and GIScience professionals may have a difficult time choosing or knowing the appropriateness of a particular tool for a particular use-case.

A growing literature base has begun extensively testing DL classifiers against traditional ML classifiers in a variety of environments and with several data types and sizes [21-24]. The goal of these studies is to identify the best performing classifier by comparing the results of the classifiers on the same datasets, usually using the same or similar training and validation data. The results of these studies have thus far been inconclusive as to which classifier (DL or ML) performs best for many environments, though the current available literature can provide guidance for professionals looking to use such tools for particular applications (e.g., land use/land cover classification, vegetation cover, coral reef habitat classification) [25-27]. Certainly, each tool should be selected based on how it best answers the research question. However, few studies have used the GUI tools developed for less-technically inclined researchers by the large GIS software companies.

The present study seeks to identify the best performing classifier (among three effective and commonly used DL and ML classifiers) for mapping land-use/land cover (LU/LC) using a large, complex county-wide dataset for a coastal county. Large, high resolution imagery datasets of coastal areas that include complex land cover can be more difficult to process and classify, depending on the research question, methods, quality of image, and field data. High resolution imagery can introduce a salt-and-pepper effect due to intraclass variations. Knowing which classifier performs best in this type of environment and with imagery of these specifications will especially benefit coastal managers and practitioners. This study will specifically use the ML and DL tools embedded in Esri ArcGIS Pro 2.8.1.

Three effective and commonly used ML and DL classifiers are compared in this study. The U-Net Convolutional Neural Network (CNN) is a DL algorithm that was originally created for biomedical image segmentation but has been now used for remote sensing image classification applications [28-29]. The architecture of the network can be divided into two halves - the first being an encoding or "contracting" side and a decoding or "expansive" side - that give the architecture its " $u$ " shape. The U-net algorithm has shown success in classifying coastal wetlands using remotely sensed imagery in previous studies [30-31]. SVM and RF classifiers are commonly used ML classifiers for remote sensing analysis. The SVM classifier is a supervised classification method based on the statistical learning theory and was developed in the computer science community in the 1990s [32] (pg. 337). It is now commonly used in remote sensing research [21,33-34]. SVM classifiers are beneficial because they can handle small training samples and the training samples do not need to be normally distributed. SVM classifiers can handle non-linear class boundaries and multiple classes. The RF classifier is a supervised classification method based on the random forest statistical method [32] (pg. 311). A series of decisions are made based on the statistical makeup of the classes and the image overall. The decisions branch out together and form what look like tree branches. When the entire image is classified, many instances of classification are performed on subsets of the data, therefore creating many decision trees [35]. The most frequent tree output is used as the overall classification. Using multiple trees is meant to mitigate overfitting to the training samples provided 
by the user. The 'best' classifier will be determined by comparing time costs for classifying the imagery and overall accuracy $(\mathrm{OA})$ results.

Upon determining the most effective classifier, a case study for demonstrating the effective use of the best classifier is conducted using the large county-wide data set to detect change in LU/LC over a ten-year period that can affect the extensive marsh environment across a large county in South Carolina, USA. While direct modification of the marsh environment for the sake of development is important to map, indirect impacts such as pollution, excessive nutrient and sewage inputs, other upstream development and freshwater diversions coming from coastal communities may have lasting negative effects on the health of our important coastal wetlands [36-38].

Section 2 of this study outlines the materials and methods of the experiment and case study. This includes a description of the study area, the data used, how classifiers were trained and applied, and change detection analysis was conducted. Section 3 presents the results from the comparison and case study. Results of this case study provide insights for coastal managers to better monitor and adaptively manage for marsh health. Finally, results are discussed in the context of the current literature base, followed by a brief conclusion.

\section{Materials and Methods}

\subsection{Study Area}

Beaufort County is one of South Carolina's populous counties nestled in the southern coast of the state (Figure 1). From 2010 to 2019, the population in Beaufort County grew from 162,233 to 192,122 , an increase of $18.4 \%$ [40]. It ranks as the wealthiest county in the state with respect to the median household income at $\$ 68,377$. Beaufort County is home to half of the state's salt marsh [41]. According to [42], South Carolina salt marshes and coastal wetlands provide services in the four ecosystem service categories: provisioning, regulating, cultural, and supporting. Each of these categories, though not all marketed services, provide valuable resources to coastal communities. For example, the salt marshes serve as nursery habitat to many species, especially shrimp. South Carolina's commercial fishing industry that relies upon these environments generates $\$ 42$ million dollars annually to the state economy [43]. Other services, like flood protection, carob sequestration, filtration, and tourism all contribute to the enormous value these marshes are to the South Carolina coast. The predominate species of marsh vegetation is Spartina Alterniflora. Juncus Romerianus is also commonly found. The county is home to a diversity of several land cover types including wetlands, forests, large water bodies, extensive housing and commercial developments, and agriculture. As the county's population continues to grow in a dynamic and complex coastal environment, the importance of monitoring change using accurate classification methods is critical for future planning and measuring trends in socioeconomic and ecological health. 

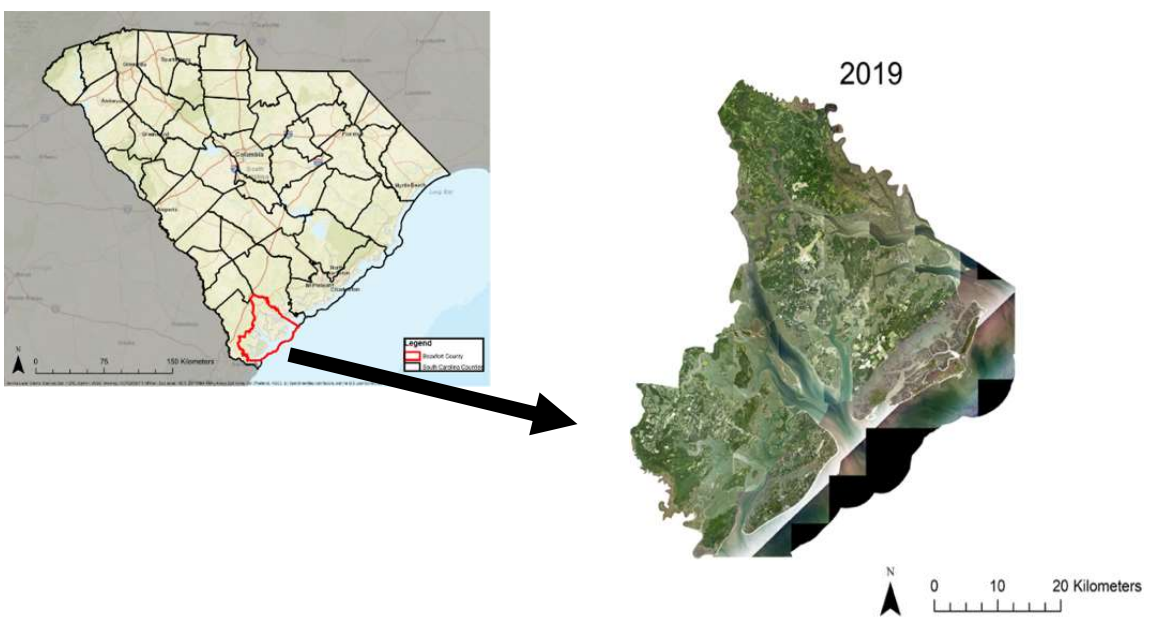

Figure 1. Map of South Carolina with Beaufort County (in red) highlighted and its NAIP image acquired in 2019.

Despite extensive regulations to abate the environmental impacts of development on Beaufort County's salt marsh, in [44] (pg. 33), community stakeholders continue to voice concerned for the health of the marsh. While no substantial evidence of marsh loss was cited, additional insights from the document state that lack of monitoring in Beaufort County is a detriment to our understanding how the marsh is being affected [44] (pg. 34). Furthermore, the aforementioned Beaufort County comprehensive plan does not address marsh migration in the face of sea level rise [45]. In addition to comparing DL algorithm competency in mapping a large, complex county wide image with other ML classifiers, this study seeks to fill a gap in the understanding of land use/land cover extent changes in the area that may be directly or indirectly impacting marsh health.

\subsection{Data}

The aerial imagery used in this study were collected by the National Agriculture Imagery Program, or NAIP. This program began in 2002 and is administered by the U.S. Department of Agriculture (USDA) Farm Service Agency to collect aerial imagery during growing seasons. The digital sensors used for NAIP imagery, though not apparent in the metadata provided with the imagery, meet rigid calibration specifications [46]. NAIP imagery is generally collected at a $1 \mathrm{~m}$ spatial resolution $(50-60 \mathrm{~cm}$ in some areas) across the conterminous United States.

For this study, NAIP images of Beaufort County acquired in 2009 and 2019 were used. The imagery varies in the month collected. For the 2009 NAIP imagery, each tile in the orthomosaic was collected between April 16 and April 25, 2009. The 2009 imagery is a traditional true color orthomosaic, with a $1 \mathrm{~m}$ spatial resolution captured by a Leica Geosystems ADS40-SH52 sensor (sensor numbers 30028 and 30045). The 2019 imagery was collected between August 29 and September 23, 2019. The 2019 flights resulted in a $60 \mathrm{~cm}$ spatial resolution and true color imagery from a Leica Geosystems ADS100 model sensor (sensor numbers 10530 and 10552). The pixel size was resampled to $1 \mathrm{~m}$ to match the 2009 image. The tide of each image varied, even within an image due to the flight times of each tile that makes up the images. In general, the 2009 image shows higher tides with much of the lower marsh slightly inundated. The National Wetland Inventory (NWI) shapefile for South Carolina was used to mask out deep water bodies while retaining marsh areas [47]. After masking, the imagery was reduced to a smaller size (about $11 \mathrm{gb}$ ) and became more manageable for classifications. Both images were transformed into the NAD83 (2011) UTM 17N coordinate system. 
Areas of interest (AOIs) for training and validation samples were manually digitized from the $1 \mathrm{~m}$ NAIP imagery based on expert knowledge in the study area and field visits within the last two years. A portion of the open-source building footprints layer provided by Microsoft was used as AOIs for the building class [48-49]. While the dataset was produced in 2018, the individual tiles or scenes throughout the imagery collected in a wide variety of dates. Therefore, a Beaufort County building footprints subset was thoroughly examined before usage as ancillary training and validation data. An accuracy assessment was performed for each classified image using the validation AOIs generated in a similar manner to the training AOIs. A stratified random sampling of 1500 validation points were generated for the validation process (Figure 2).

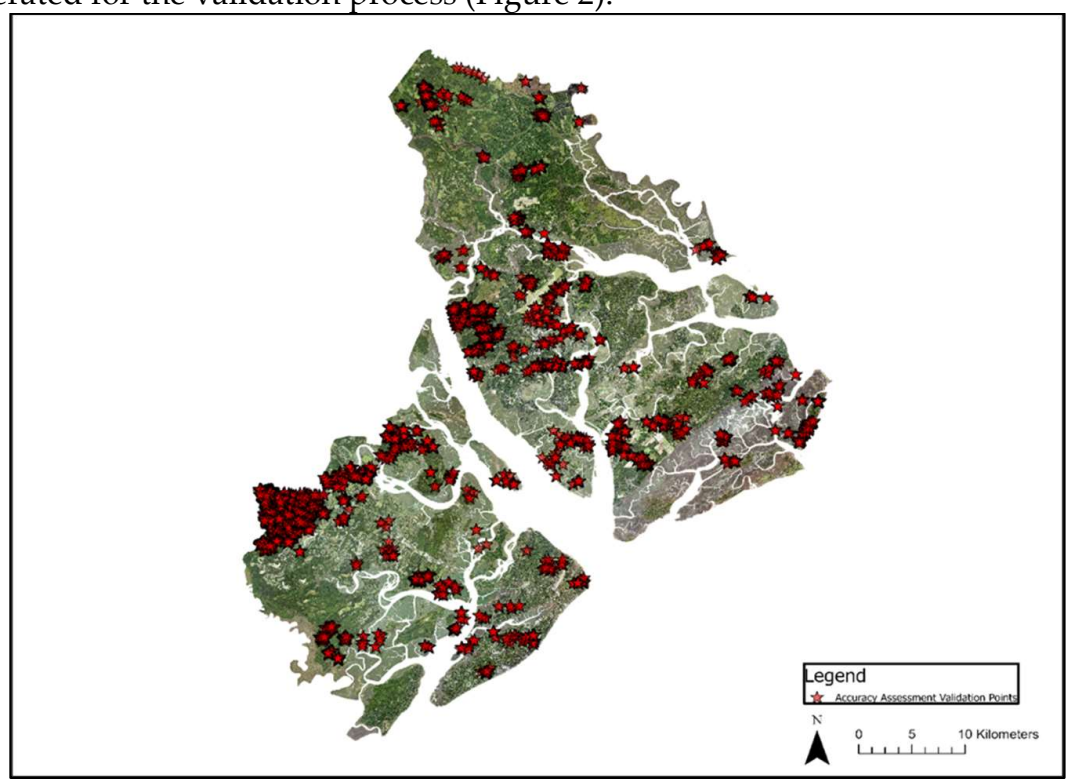

Figure 2. Distribution of sample points used for accuracy assessment across Beaufort County.

\subsection{LU/LC and Classification Methods}

A general workflow of this study is represented in Figure 3. Both NAIP images were classified into several level 1, 2, and 3 LU/LC classes, loosely based on [50]. The level 3 classes included mudflat, marsh vegetation, forest, roads, buildings, agriculture, grassland, water, shadows, dry bare ground and wet bare ground (Table 1). Several classes were combined in our level 2 classes to leave 7 predominant classes that described the general LU/LC in the study area. For example, mudflat and marsh vegetation were combined into the marsh class, roads and buildings into the urban class, and dry and wet bare ground into a single bare ground class. It was determined that the agriculture class and grass classes were significantly confused, and therefore were combined due to their similarities. These combinations were made to identify general environments and limit unnecessary misclassifications.

Table 1. Classes used in this study. The dashes indicate the previous class is now included in the

\begin{tabular}{ccc}
\hline Level 3 Class & Level 2 Class & Level 1 Class \\
\hline Mudflat & Marsh & Marsh \\
Marsh Vegetation & Marsh & Marsh \\
Forest & Forest & Forest \\
Roads & Urban & Development \\
Buildings & Urban & Development \\
Agriculture & Ag/Grass & Development \\
Grassland & Ag/Grass & Development \\
\hline
\end{tabular}




\begin{tabular}{ccc}
\hline Dry Bare Ground & Bare Ground & Development \\
Wet Bare Ground & Bare Ground & Development \\
Water & Water & Water \\
Shadows & Shadows & Shadows \\
\hline
\end{tabular}

A final set of level 1 classes were determined by combining bare ground, urban, and grass/agriculture into a developed class that was used as a proxy for general LU/LC changes that can impact marsh health. Bare ground is included in development because construction sites and pre-construction sites across the county are typically bare ground. Grass is included as a development class because it represents a loss of natural forested coastal area. Many parks and yards are part of developments, and this is where the grass is found. Further, while agricultural use may be impactful if the farmer is using certain chemicals, the same could be said for large grass areas where added nutrients can eventually reach the wetlands through runoff. Other classes, like water, shadows, forest and marsh, remained separate in the level 1 classification. In the end, the forest and development classes were deemed the most important for determining changes that would affect marshes. Aside from determining the actual marsh changes (i.e., development on marsh or marsh gain through marsh restoration), the changes in forested land and increase in development were used as an indicator of how marsh may be affected.

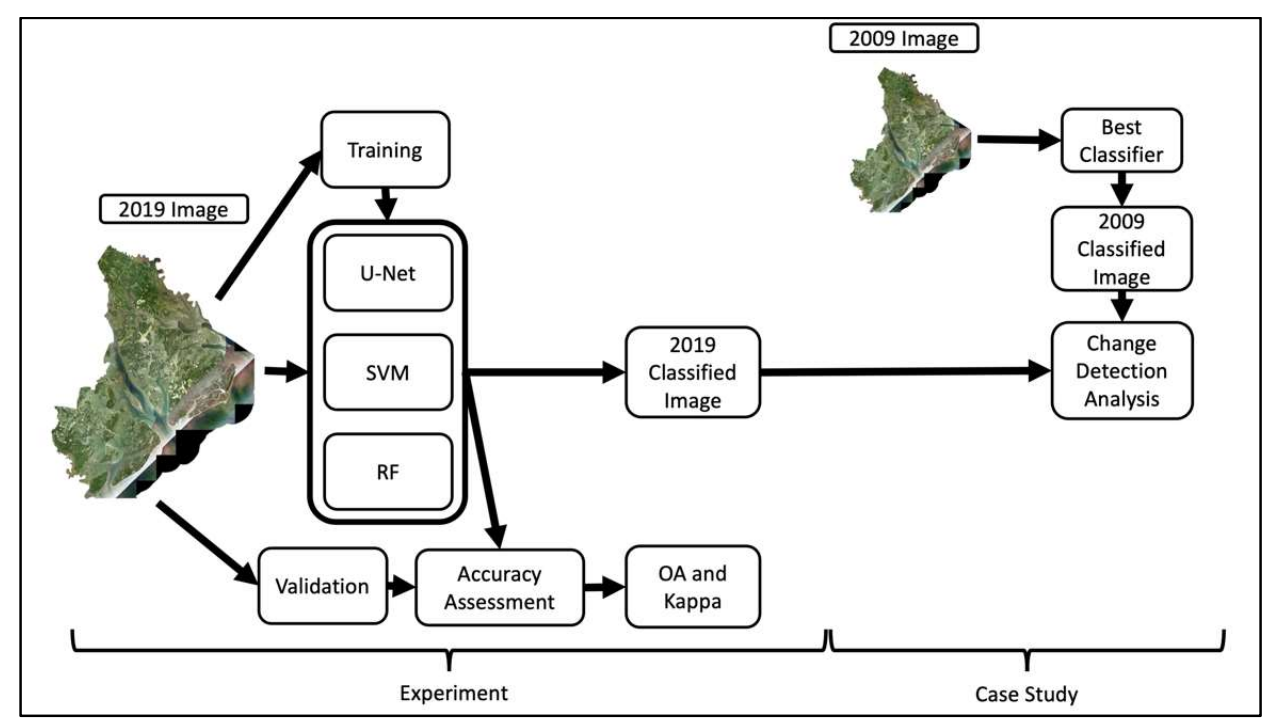

Figure 3. A general workflow for the experiment and case study. First, training data was used to classify the 2019 image using the three different classifiers (U-Net, SVM, and RF). Accuracy assessment was used to determine the most effective classifier. The 2009 image was classified using the best performing classifier and then compared with the 2019 image for change detection analysis.

Object oriented classifiers (OOC) were used for these classifications because of their ability to mitigate some of the high-resolution intra-class detail and salt-and-pepper phenomenon that often occurs with pixel-based classifications [51]. While noise can still be found in the objects, object parameters can smooth out much of the salt-and-pepper effect. OOC have been shown to have better accuracy than pixel-based classifiers in a variety of environments, including salt marsh and other LU/LC classes found in our study area [5254]. The U-net classifier performed the semantic segmentation without the user input of a segmented image. For the SVM and RF classifications, the base image was segmented using different properties. Spectral detail was placed at the highest importance, with spatial detail coming in second. On the range of 1.0 to 20.0 , spectral detail was placed at 18.5, 
while spatial detail was placed at an eight in an effort to smooth out the image. Minimum segment size was placed at 5 pixels $\left(5^{*} 5 \mathrm{~m}\right)$ so as to accommodate smaller buildings and small patches of marsh vegetation.

Each classifier required certain input parameters beyond the images and training data. To train the U-Net DL classifier, the training AOIs were used as inputs into ArcGIS pro's Train Deep Learning Model tool. During this process, the entire raster image was segmented into 7,169 tiles with the dimensions of $256 * 256$ pixels. The training data were embedded as well. Once the classifier was trained using the training data, all of the small tiles were input into the U-net classifier and ultimately classified one at a time before being mosaicked together again to create the whole classified image. The final output was a classified image raster.

The SVM classifier required the input of the segmented image, training samples, and classification scheme. Only a single parameter of 500 maximum samples per class was required, which limits the number of training samples you can use for each class. The parameter was set to the default given by ArcGIS Pro 2.8.1. Once each of the inputs were collectively used to train the SVM classifier, it was applied to the entire county-wide image. The final output was a classified image raster.

The RF classification followed a similar method. The same inputs were required to train the RF, though the required parameters were different. The RF classifier was trained using the following parameters: 120 maximum trees, maximum tree depth of 30, and 1000 as the maximum number of samples per class. Each of these parameters limited the size of the forest during the classifier training, while seeking to maintain a high level of accuracy. Once the classifier was trained it was applied to the entire county-wide image. The final result was a classified image raster.

The 2009 imagery was classified in a similar manner to the 2019 imagery. Training samples were gathered visually from the imagery in collaboration with the Beaufort County Mapping and Applications Director, who has had residence in the position since 1995. Due to the higher tide levels in the 2009 image, a new class 'underwater marsh' was included to potentially capture more marsh accurately in the image. The underwater marsh was later merged with marsh vegetation and mudflat into the level 2 and level 1 marsh classes.

\subsection{Accuracy Assessment}

A confusion matrix was calculated using ArcGIS Pro's Compute Confusion Matrix tool, where the Producer's accuracy, User's accuracy, Overall Accuracy, and Kappa were computed. Producer's accuracy is the total number of pixels classified correctly for a class divided by the total number of pixels in that class as determined from the ground truthing data. User's accuracy is the total number of pixels correctly classified into a class divided by the total number of pixels classified into that class. An overall accuracy (OA) percentage was also calculated:

$$
\mathrm{OA}=\frac{\sum_{i=1}^{K} x_{i i}}{N}
$$

where $x_{i i}$ represents a pixel classified correctly, and $N$ is the total number of pixels being assessed.

Kappa analysis is a multivariate technique for accuracy assessment first published in a remote sensing journal in 1983 [55]. Kappa is similar to overall accuracy as a measure of the accuracy of the entire classification, but each considers slightly different information. A kappa estimate $(\widehat{K})$ was determined as [56]:

$$
\widehat{K}=\frac{N \sum_{i=1}^{K} x_{i i}-\sum_{i=1}^{k}\left(x_{i+} \times x_{+j}\right)}{N^{2}-\sum_{i=1}^{k}\left(x_{i+} \times x_{+j}\right)}
$$

where $N$ is the total number of samples, $k$ is the number of rows in the confusion matrix, $x_{i i}$ is the number of observations in row $i$ and column $i$, and $x_{i+}$ and $x_{+j}$ are the marginal totals for row $i$ and column $j$. 


\subsection{Case Study - Change Detection and Marsh Analysis}

Following the classification of both the 2019 and 2009 images of Beaufort County, a change detection analysis was conducted using the Change Detection tool in ArcGIS pro v.2.8.1. The tool requires an input of a series of maps or images and computes a change detection map and change matrix in return. For the change detection analysis, a final classification map with combined classes was created. Water, shadows, marsh, and forest classes remained intact, but the agriculture/grass, bare ground, and urban classes were combined into a class called development. Areas of change were assessed based on the numbers of pixels that changed from a particular class to another. Pixel counts were multiplied by the $1 \mathrm{~m} * 1 \mathrm{~m}$ pixel size to determine approximate area in meters squared. Further conversion from $\mathrm{m}^{2}$ to ha was accomplished by multiplying by 0.0001 .

While mapping a marsh class alone gives us direct information on actual changes in the marsh, many indirect impacts from nearby land use/land cover changes have been documented [37, 57]. Because of these documented impacts, we decided to map all classes to suggest and discuss what changes may potentially occur if development trends continue.

Pixels that changed from any particular class to the shadow class, or from the shadow class to another class were disregarded for this change analysis. The pixels of interest for this study were the pixels that changed from the marsh class or the forest class to any other class, but particularly to the development class. The pixels that experienced these changes were mapped and visually analyzed to determine impacts and assess potential future impacts.

\section{Results}

\subsection{Comparison of model performance and accuracies}

Processing time is an important factor in processing large-size imagery. Computational costs depend on the data being processed as well as the computational abilities of the machine being used. Here, a Dell Inspiron 5680 6-core intel i7 CPU with 16gb Ram and Nvidia GTX 1060 3gb GPU was used to process each classification. As noted in Table 1, the U-Net classifier required the least training time but the most total classification time to apply the trained model to the image. Training the U-net classifier required 2 hours and 59 minutes, at least 30 minutes faster than the two other classifiers. However, the classification of the image itself took 43 hours and 23 minutes for a total of 46 hours and 22 minutes. The length of classification is not a common finding, however, as U-net classifiers have been found to be faster than many others in remote sensing applications [30-31, 58]. The authors suggest the extra length of time required to complete classification was due to the machine specifications, the size of the dataset, and the methods by which the tiles were classified and subsequently mosaicked together. The SVM classifier required 4 hours 52 minutes for training and then 30 minutes to classify the image. The RF classifier was trained in 4 hours 29 minutes and was applied to the image in approximately 23 minutes. The computational times and classification accuracies are reported in Table 2.

Table 2. Computational time and classification accuracies.

\begin{tabular}{ccccc}
\hline Classifier & Training Time & Classification Time & OA & Kappa \\
\hline 2019 DL U-Net & 2 hours, 59 minutes & 43 hours, 23 minutes & $92.4 \%$ & $89.8 \%$ \\
2019 SVM & 4 hours, 52 minutes & 30 minutes & $81.6 \%$ & $75.3 \%$ \\
2019 RF & 4 hours, 29 minutes & 23 minutes & $75.7 \%$ & $67.3 \%$ \\
2009 DL U-Net & 2 hours, 46 minutes & 42 hours, 34 minutes & $85.3 \%$ & $80.5 \%$ \\
\hline
\end{tabular}

The overall accuracies of the three classifiers for the 2019 image ranged from $75.74 \%$ to $92.38 \%$, with the U-Net classifier performing the best (Table 2). This study found the 
object-based ML classifiers did not perform as well, though certain classes performed well (Tables 3, 4 and 5). The forest class was consistently classified with high users and producers' accuracy (99-89\%). Other classes varied based on the classifier. For example, the SVM and RT classifiers correctly classified marsh at least $70 \%$ of the time, though the Unet Classifier had a user's accuracy of $84.41 \%$ and a producer's accuracy of $93.52 \%$. However, both ML classifiers interestingly confused marsh with the urban class the most. It is proposed that the high-resolution imagery and complex environment lead to a high intraclass variability, making it difficult for the ML classifiers to separate the classes.

Table 3. Accuracy for the predominant Level 2 classes (U-net)

\begin{tabular}{cccccccc}
\hline Classes & Marsh & Forest & Urban & Agriculture/Grass & Bare Ground & Total & $\begin{array}{c}\text { Users } \\
\text { Accuracy }\end{array}$ \\
\hline Marsh & 260 & 2 & 8 & 2 & 28 & 308 & $84.42 \%$ \\
Forest & 2 & 592 & 1 & 0 & 0 & 596 & $99.33 \%$ \\
Urban & 0 & 0 & 283 & 0 & 10 & 294 & $96.26 \%$ \\
Agriculture/Grass & 6 & 12 & 11 & 123 & 3 & 156 & $78.85 \%$ \\
Bare Ground & 10 & 0 & 0 & 3 & 84 & 97 & $86.60 \%$ \\
Total & 278 & 608 & 307 & 129 & 125 & 1509 & \\
Producer's & $93.53 \%$ & $97.37 \%$ & $92.18 \%$ & $95.35 \%$ & $67.20 \%$ & & \\
Accuracy & & & & & & & \\
\hline
\end{tabular}

Table 4. Accuracy for the predominant Level 2 classes (SVM)

\begin{tabular}{cccccccc}
\hline Classes & Marsh & Forest & Urban & Agriculture/Grass & Bare Ground & Total & $\begin{array}{c}\text { Users } \\
\text { Accuracy }\end{array}$ \\
\hline Marsh & 217 & 6 & 10 & 1 & 19 & 263 & $82.51 \%$ \\
Forest & 0 & 561 & 1 & 1 & 1 & 567 & $98.94 \%$ \\
Urban & 37 & 7 & 273 & 11 & 63 & 400 & $68.25 \%$ \\
Agriculture/Grass & 22 & 9 & 10 & 111 & 10 & 167 & $66.47 \%$ \\
Bare Ground & 2 & 0 & 9 & 5 & 32 & 49 & $65.31 \%$ \\
Total & 278 & 608 & 307 & 129 & 125 & 1509 & \\
Producer's & $78.06 \%$ & $92.27 \%$ & $88.93 \% \%$ & $86.05 \%$ & $25.60 \%$ & & \\
Accuracy & & & & & & & \\
\hline
\end{tabular}

Table 5. Accuracy for the predominant Level 2 classes (RF)

\begin{tabular}{cccccccc}
\hline Classes & Marsh & Forest & Urban & Agriculture/Grass & Bare Ground & Total & $\begin{array}{c}\text { Users } \\
\text { Accuracy }\end{array}$ \\
\hline Marsh & 201 & 19 & 15 & 4 & 11 & 260 & $77.31 \%$ \\
Forest & 2 & 541 & 12 & 6 & 0 & 573 & $94.42 \%$ \\
Urban & 55 & 23 & 265 & 0 & 73 & 448 & $59.15 \%$ \\
Agriculture/Grass & 8 & 24 & 10 & 105 & 0 & 152 & $69.08 \%$ \\
Bare Ground & 12 & 0 & 5 & 14 & 41 & 72 & $56.94 \%$ \\
Total & 278 & 608 & 307 & 129 & 125 & 1509 & \\
Producer's & $72.30 \%$ & $88.98 \%$ & $86.32 \%$ & $81.40 \%$ & $32.80 \%$ & & \\
Accuracy & & & & & & & \\
\hline
\end{tabular}

After finding the U-Net classifier performed the best, it was applied to the 2009 NAIP dataset as well. Overall accuracy of the 2009 image classification was $85.28 \%$, with a Kappa statistic of $80.45 \%$. While many of the same classes performed remarkably well between the two sets of imagery, the tidal ranges within the 2009 image seems to have proved difficult for the U-net classifier. The producer's accuracy for the marsh class was a low $65.18 \%$, though the user's accuracy was $96.90 \%$. The marsh areas were often confused for 
the water class or the agriculture/grass class due to the high tides and time of year. The agriculture/grass class was often confused as well. It is suggested that this was due to the image collection during peak biomass, when $S$. Alterniflora is its greenest and most like an agricultural product or grass. Outside of the marsh class and the bare ground class that was confused for urban areas, all other classes resulted in a producer's accuracy of at least $90.0 \%$.

\subsection{Comparison of classification results}

The DL U-Net classifier was able to navigate the complexities of the environment better than the other ML classifiers and achieved a higher accuracy. Evidence of this assertion can be found in three subset areas with classification challenges. Figure 4 shows a forested and agricultural area in northern Beaufort County. It is classified reasonably well by U-Net (Fig. 4A), which shows very little salt-and pepper effect in the classification. However, SVM and RF (Fig. 4B-C) misclassified the forest as water, marsh or shadow depending on the hue of the green space. Portions of the marsh on the western edge of the image were confused for bare ground by RF, though the U-net and SVM classifiers generally recognized it to be marsh. SVM also misclassified small portions of the marsh area as urban area. U-Net struggled with wet areas in and around inland water bodies, classifying surrounding vegetation as marsh. 


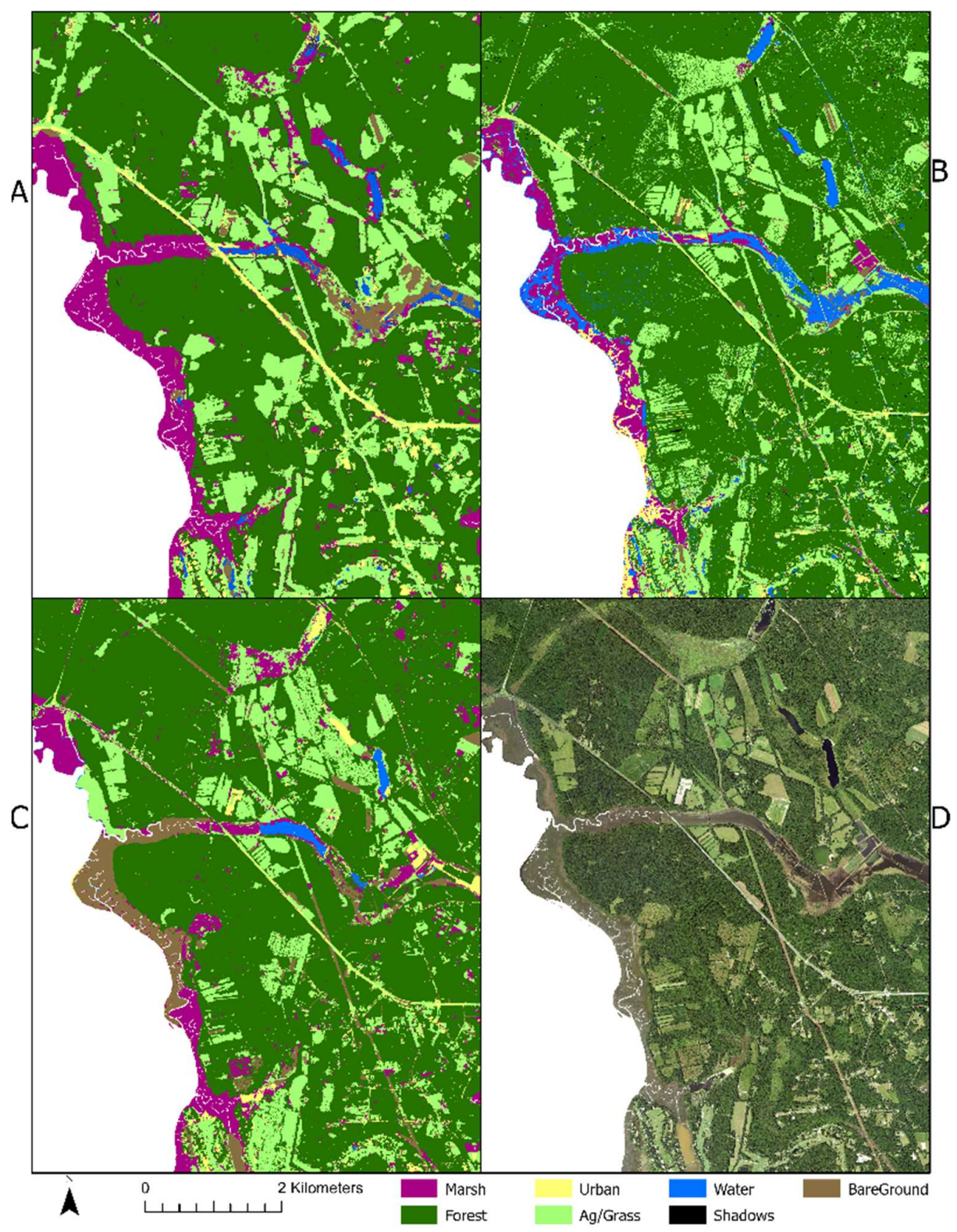

Figure 4. A mixed-use area classified by A) U-net, B) SVM and C) Random Forest. D) is the NAIP image of this subset.

In another subset area under development (Figure 5), U-Net (Fig.7A) once again classifies the bare ground areas correctly, along with the extensive suburban areas. SVM similarly classified most of the bare ground and urban areas correctly. However, RF misclassified the bare ground areas as urban areas. Another difficulty for each classifier was differentiating some wetland areas and ponds in neighborhoods and golf courses from the marsh. SVM and U-Net occasionally misclassified those areas, while RF struggled the most. Nearly every inland water body was misclassified as marsh by the RF classifier. 


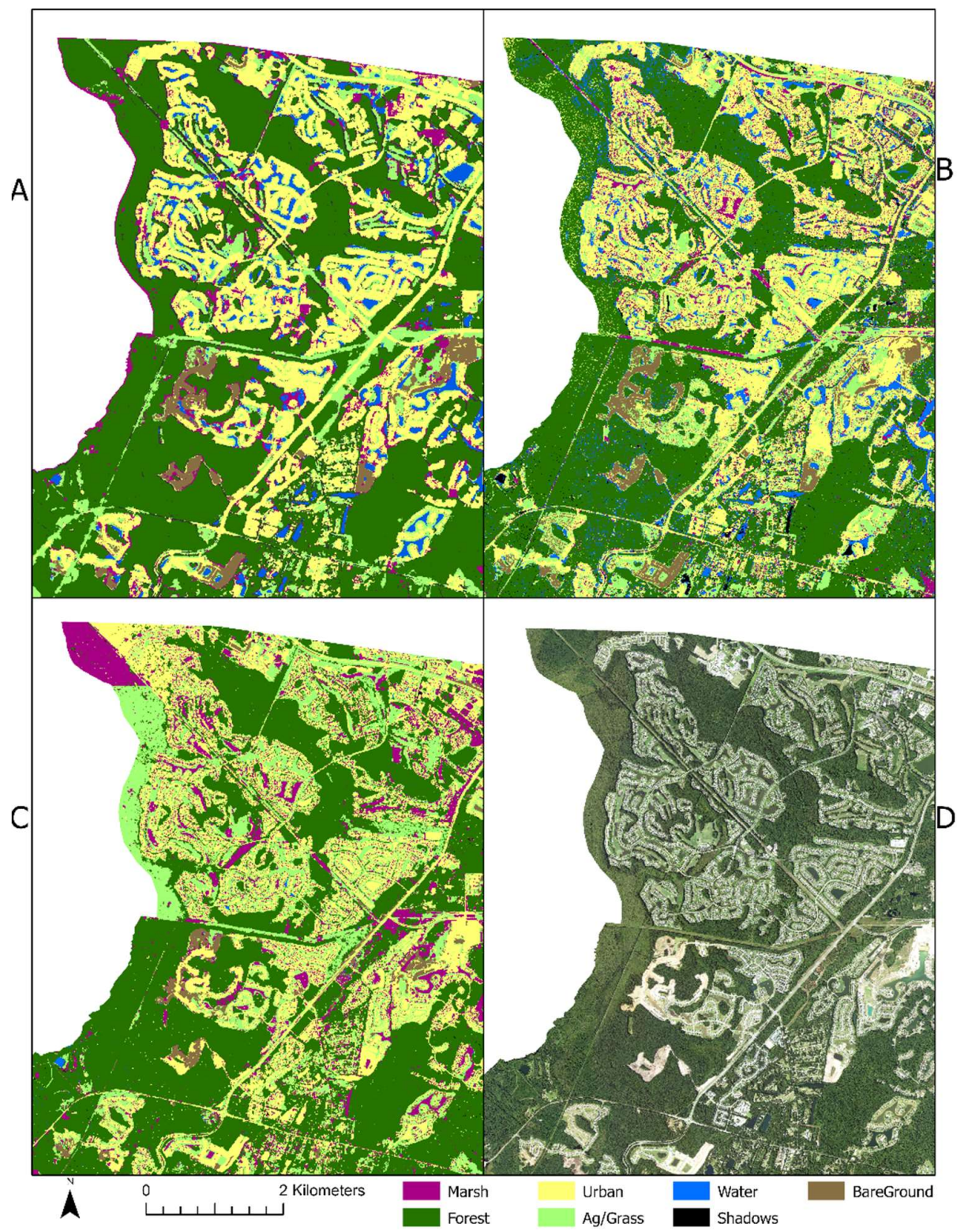

Figure 5. A developing area classified by A) U-net, B) SVM, and C) Random Forest. D) is the NAIP image of this subset.

It is well known that marsh extent is difficult to map, especially when the tidal range varies throughout the imagery. These tidal discrepancies made classifying the marsh difficult for each classifier in this study (Figure 6). Marsh was sometimes misclassified as water, urban, bare ground, and even grass. If images were collected during low tide conditions across the entire study area, classifications could have been more accurate. Water hues ranging from blue to algae-ridden green waters made classifications of water and grass difficult as well. Ancillary information, such as texture, RGB-based indices, or even a DEM might assist in the differentiation between some of the more troubled classes. Some inland areas in and around small ponds were also misclassified as marsh. This is similar to RF that struggled to differentiate the water class from the marsh class in nearly every inland pond (as shown in Figure 5C). 


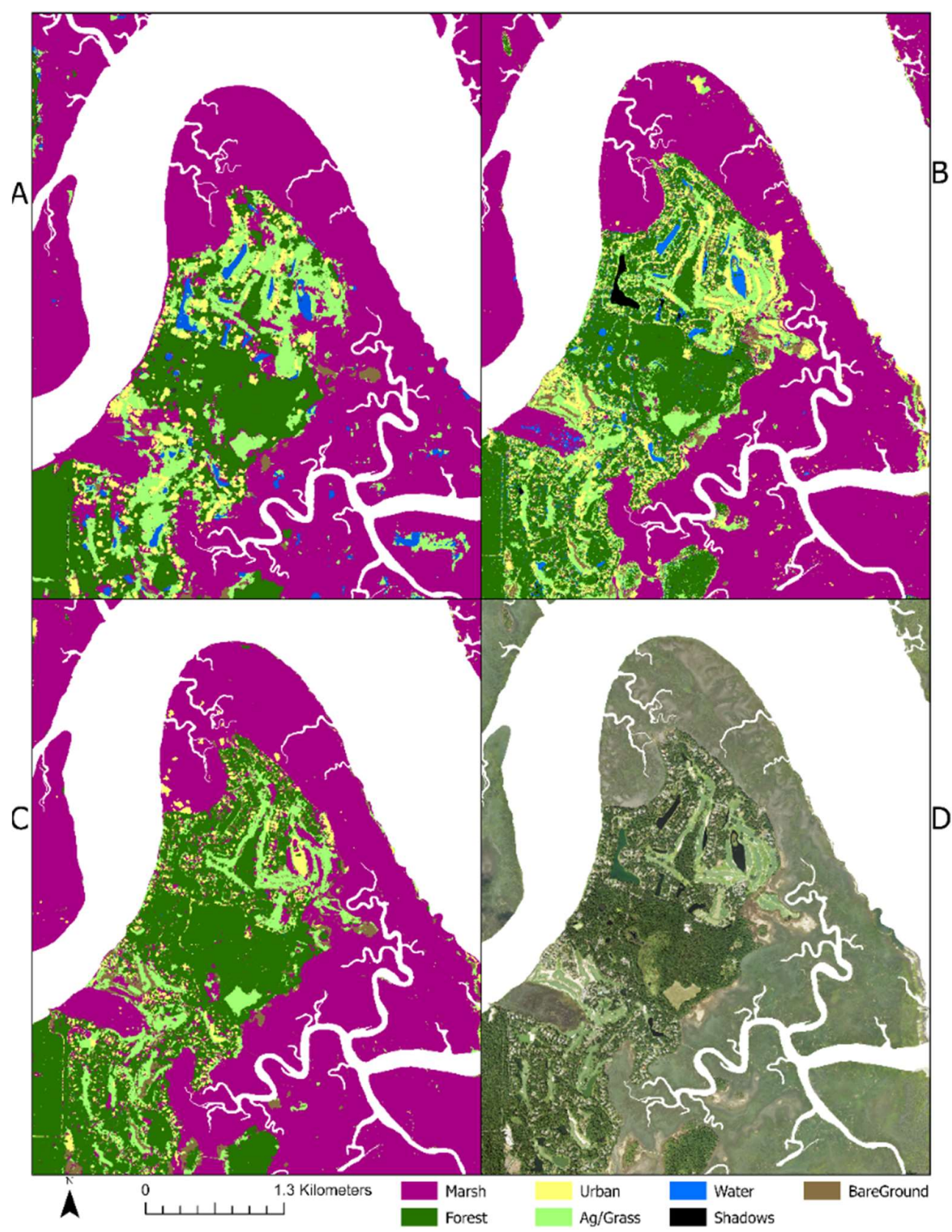

Figure 6. A marsh area classified by A) U-net, B) SVM, and C) Random Forest. D) is the NAIP image of this subset.

\subsection{Coastal Development and Impact to Marshes}

After the DL U-net classifier was applied to both the 2019 and 2009 images, changes between the two dates were assessed visually and, as best as possible, quantitatively. The 2009 image classification struggled to classify marsh correctly in some areas, assigning some pixels as development (i.e., the urban, grass/agriculture, or bare ground classes) rather than marsh. With this knowledge, it is apparent that several areas that were marked as marsh loss or gain were in fact errors made by the classifier. These areas were visually inspected. Figures 7C and 7D indicate two areas where actual changes did occur, and in fact some marsh vegetation was lost. An overall marsh system loss was be estimated at 3,300 ha. However, because of the errors detected extensively throughout the 2009 marsh class in particular, a quantitative assessment of marsh losses may not be completely trusted. To reiterate the issues described above, the producer's accuracy of the marsh class 
in the 2009 image was only $65 \%$, the lowest of all the classes. The marsh was misclassified as urban area because of sun glint, and sometimes as agriculture due to its greenness. We used expert visual observation to determine that there was very little true marsh loss over the ten-year period. There were a few areas where marsh vegetation extent expanded between dates (Figure 7A and 7B), but there were no detected areas where the marsh vegetation or mudflat were directly affected by development in the marsh system. Tidal levels throughout both images made classifications and comparisons difficult. Despite using a separate class for submerged or underwater marsh, these areas are where much of the misclassifications occurred among the marsh class.

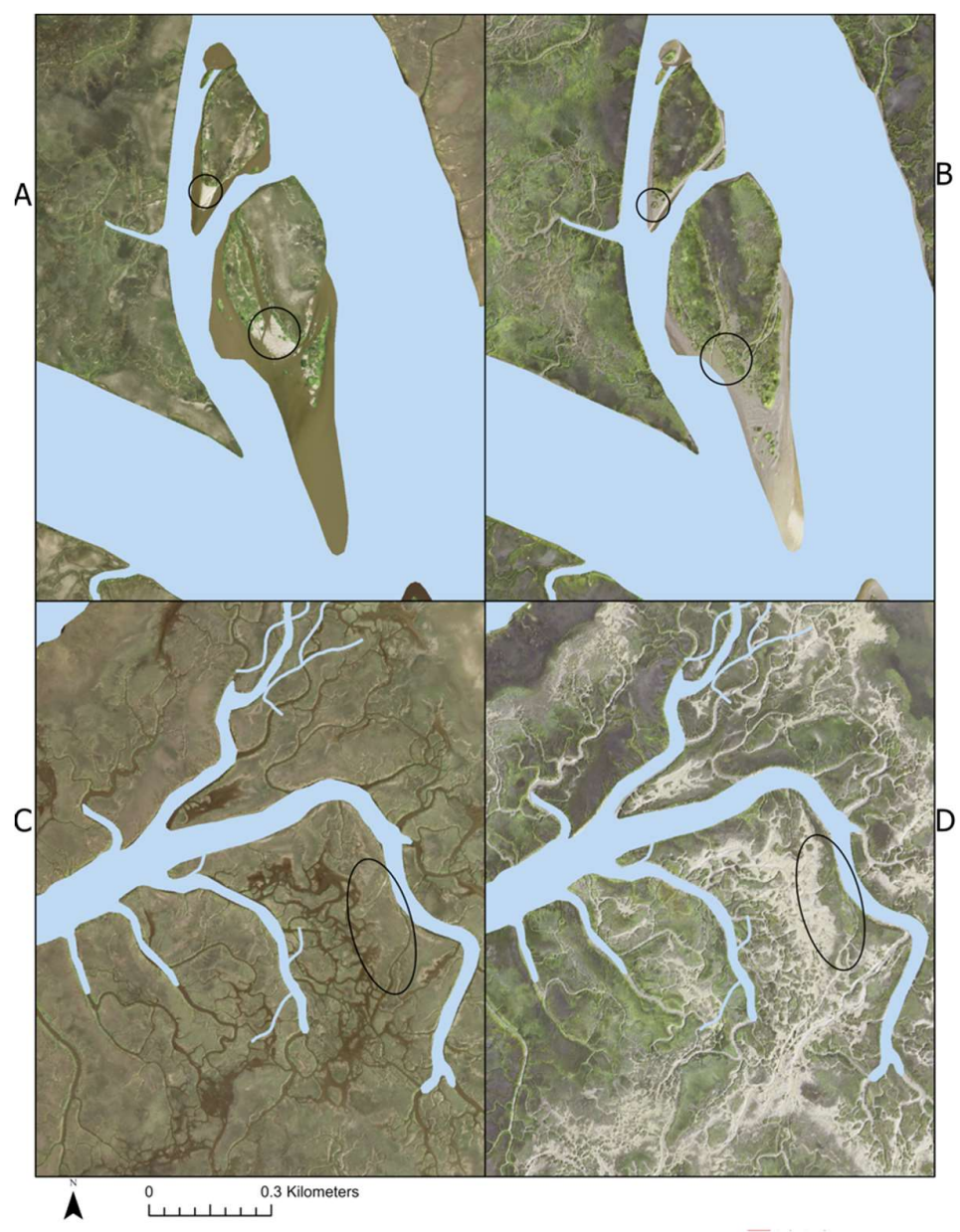

Figure 7. Examples of marsh vegetation gain (A and B) and loss (C and D) from 2009 to 2019. 
Large areas of development expansion were detected across the county. This study indicates that approximately 7,102.74 ha of forest were lost to other land cover classes (e.g., urban, bare ground, and grass/agriculture). For the purposes of the case study, any forest lost to the level 1 development class was deemed development. The development in northern Beaufort County included small areas of urban development and large areas of agricultural development. Southern Beaufort County saw the greatest amount of urban growth. Figure 8 indicates the area of development across the county. On the other hand, some previously urban areas from 2009 were naturalized over the past 10 years. Some agricultural and urban areas from 2009 were overtaken by shrubs and small trees over the ten-year period. These areas were often then classified as forest and were counted as lost developed land.

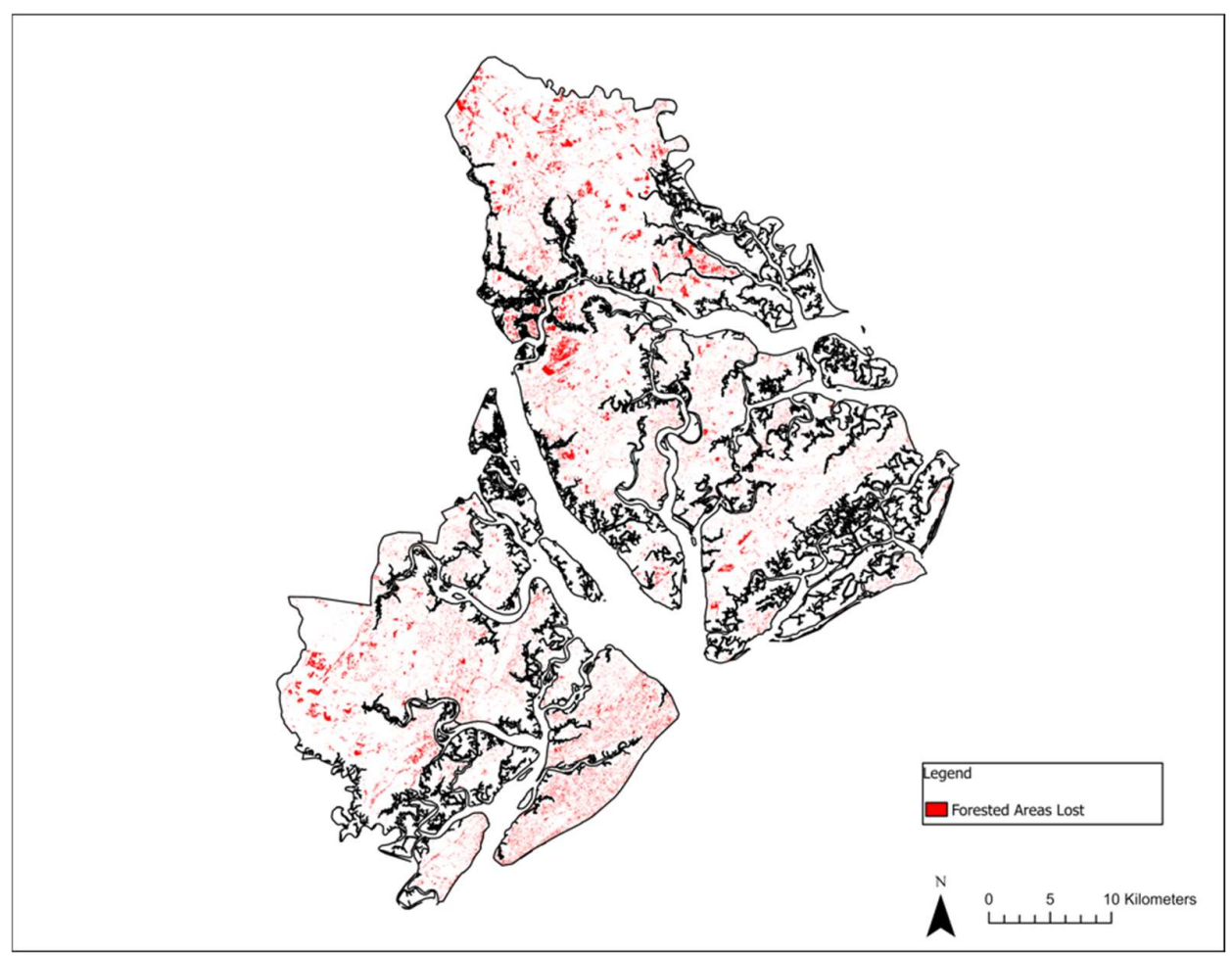

Figure 8. Forested areas lost to development across Beaufort County in 2009-2019.

\section{Discussion}

The DL, SVM, and RT classification results fared well when compared to other largearea DL mapping studies that included wetlands and other complex land cover classes. For large scale wetland mapping across Alberta Canada, [59] achieved an 80.2\% OA using a deep CNN. In a study comparing RF, SVM, and three other deep learning classifiers for classifying wetland using small unmanned aerial systems hyperspatial resolution imagery, [60] found that the DL classifiers performed better than the SVM and RF classifiers, especially when the training sample counts were high. RF and SVM classifiers resulted in OA as high as $65 \%$ and $67 \%$, respectively. The DL classifiers resulted in OA upwards of $76 \%$ to $84 \%$. Similarly, our results support assertions made by [61] that CNN can outperform RF classifiers. Specifically, U-Net has shown to outperform SVM and RF classifications for wetland mapping using Sentinel-2 $10 \mathrm{~m}$ imagery. [31] discovered that the SVM and RT classifiers only achieved an OA of 50.5\% and $46.4 \%$, respectively, while the U-Net classifier regularly reached at least $85 \%$, depending on the optimizing function used. Our study suggests the higher resolution NAIP imagery includes enough spatial detail to improve the OA to the detected levels of accuracy (e.g., U-Net $=92.4 \%$; SVM = 81.6; RT = $75.7 \%$ ). When using a similar spatial resolution data from the Worldview 3 satellite to 
classify forested wetlands using a DL CNN, [62] found similar accuracy levels as our study $(92 \%)$ when only the optical imagery was used.

Several challenges were faced when classifying the coastal tidal marsh in Beaufort County for this study. Maneuvering the tide, and water levels in general, is a significant challenge when using remotely sensed imagery to map coastal wetlands, including coastal tidal marshes [63]. This was particularly evident in this study, as event within the NAIP imagery for one county there was a significant difference across the tiles that made up the image in tide levels. This was one of the major difficulties in classifying the marsh. Other environmental conditions such as cloud cover and shadows cast by tall objects (like buildings, trees, and water towers) obscured the target wetlands, complicated spectral signatures, and made optical imagery difficult to interpret or use [64]. Plant phenology also played a factor in image classification. Peak biomass conditions are best for modeling plant health characteristics such as biomass and can be beneficial in mapping certain coastal wetland species [65-66]. The 2009 imagery was taken in April, which is at the beginning stages of growth and greening up for S. Alterniflora, the dominant marsh grass in Beaufort County. The 2019 imagery was taken in late august and early September, which is in the peak biomass for S. Alterniflora [67]. All three classifiers were more successful at classifying the marsh class in the 2019 imagery than the U-net classifier was in the 2009 image. We propose that plant phenology, along with tide levels throughout each image, was a significant factor in these results.

NAIP datasets provide high resolution aerial imagery with great potential for vegetation mapping, in particular when acquired during leaf-on conditions. While we found a fair amount of success mapping various classes, including the marsh class, the NAIP RGB imagery alone was not sufficient to overcome all of the complexities of the coastal wetland environment. To better classify the coastal tidal marsh, particularly the vegetation and mudflat, it would be expedient to incorporate ancillary remote sensing data. This process, called data fusion, can be used to better describe and classify wetlands [68]. Data fusion can be performed at the pixel-level, feature-level, and decision-level. [69], as described in [70], found that they could improve land cover classification by fusing multispectral data with radar data. While the increase in overall accuracy (OA) was small, some sub classes improved while others decreased slightly in classification accuracy. [71] applied a fusion of multispectral imagery with LiDAR-derived elevation datasets to map peatlands in Canada with a $76.4 \%$ OA opposed to only achieving a $65.8 \%$ with the RGB and IR bands. Data fusion is able to provide better information for decision makers. For example, the addition of a NIR band or a vegetation index such as the Normalized Difference Vegetation Index (NDVI) provides greater discrimination between marsh vegetation and mudflat, as well as marsh vegetation from other vegetation classes. Elevation data derived from LiDAR or other sources improves feature extraction of trees, agriculture, and grasses from the marsh grasses and even mudflats.

Potential biases and errors introduced in the study may be introduced in the selection of training and validation AOIs by the researchers. Potential bias was mitigated by involving multiple long-term residents of the county who interpreted the aerial imagery and selected the training and validation polygons based on extensive local knowledge.

Future work should incorporate ancillary remotely sensed data into the classification process to further increase classification accuracy. As previously stated, other spectral bands and indices, elevation data, and imagery from other scales (i.e., small unmanned aerial systems) should be examined to produce a data fusion product of potentially higher accuracy. Results from LU/LC classifications can be used as input into models for other phenomenon, like water quality [72]. Water quality is another element that can impact marsh health. Further trials with other available deep learning pixel classifiers, such as DeepLabv3, are useful tools to be investigated as well. These methods could be further validated through application and testing in similar coastal environments.

\section{Conclusions}


This study compared DL with traditional ML classifiers based on the classification of high-resolution imagery over an entire coastal county using GUI applications from ArcGIS Pro 2.8.1. Our case study then used the LU/LC maps from 2019 and 2009 to detect salt marsh change patterns over a 10-year period. Results indicated that a U-Net DL classifier significantly outperformed the other classifiers for the classification of a complex, high resolution county-wide dataset in terms of OA $(92.4 \%$ as opposed to the $81.6 \%$ by SVM and $75.7 \%$ by RF). DL algorithms now available to any coastal manager or GIS analyst with access to Esri's ArcGIS pro showed their high applicability to large-area mapping. Using computational resources commonly available to coastal managers and professional GIS analysts, the U-net classification required a longer time to classify the large dataset (46 total hours vs 5.33 hours and 4.83 hours). Because this was not found among other literature regarding other U-Net classifiers, we believe the time required for classification was a function of the large dataset, computational resources, and DL model structure. Our study focused on DL and ML classifiers from the perspective of the environmental or coastal manager. Findings indicate a bright future for DL and ML LU/LC classification for large-area mapping, even for those without complicated programming and DL or ML backgrounds. Our case study demonstrated the power of using these tools for change detection, showing large areas of development over a 10-year period across the county that may have an impact on marsh health. Further research is needed to validate findings and test similar methods across similar complex coastal environments. Additional ancillary remote sensing data, including multispectral and hyperspectral imagery, LiDAR, and RADAR, can be integrated to improve classification accuracy.

Author Contributions: Conceptualization, G.M., C.W., and Z.L.; methodology, G.M., C.W., and Z.L; software, G.M..; validation, G.M., D.M.; formal analysis, G.M.; investigation, G.M., C.W., and Z.L; resources, G.M. and D.M.; data curation, G.M.; writing - original draft preparation, G.M.; writingreview and editing, G.M., C.W., D.M, S.S., and Z.L.; visualization, G.M.; supervision, Z.L., C.W., S.S. All authors have read and agreed to the published version of the manuscript.

Funding: This research received no external funding.

Data Availability Statement: The NAIP data presented in this study are openly available in EDI Data Portal at https://datagateway.nrcs.usda.gov/GDGHome_DirectDownLoad.aspx.

Acknowledgments: The authors would like to thank USDA and the NAIP program for providing excellent imagery, and the Beaufort County Mapping and Applications department for their insightful comments.

Conflicts of Interest: The authors declare no conflict of interest.

\section{References}

1. Camps-Valls, G. Machine learning in Remote Sensing Data Processing. 2009 IEEE International Workshop on Machine Learning for Signal Processing 2009.

2. Lary, D. J.; Alavi, A. H.; Gandomi, A. H.; Walker, A. L. Machine learning in geosciences and remote sensing. Geoscience Frontiers 2016, 7, 3-10.

3. Maxwell, A. E.; Warner, T. A.; Fang, F. Implementation of machine-learning classification in Remote Sensing: An applied review. International Journal of Remote Sensing 2018, 39, 2784-2817.

4. Shang, X.; Chisholm, L. A. Classification of Australian native forest species using hyperspectral remote sensing and machinelearning classification algorithms. IEEE Journal of Selected Topics in Applied Earth Observations and Remote Sensing 2014, 7, 24812489.

5. Hänsch, R.; Schulz, K.; Sörgel, U. Machine learning methods for Remote Sensing Applications: An overview. Earth Resources and Environmental Remote Sensing/GIS Applications IX 2018.

6. Peña, J.; Gutiérrez, P.; Hervás-Martínez, C.; Six, J.; Plant, R.; López-Granados, F. Object-based image classification of summer crops with machine learning methods. Remote Sensing 2014, 6, 5019-5041.

7. Brovelli, M. A.; Sun, Y.; Yordanov, V. Monitoring Forest change in the Amazon using multi-temporal remote sensing data and Machine Learning Classification on google earth engine. ISPRS International Journal of Geo-Information 2020, 9, 580. 
8. Pal, M.; Mather, P. M. Support vector machines for classification in remote sensing. International Journal of Remote Sensing 2005, 26, 1007-1011.

9. Train Support Vector Machine classifier (spatial analyst) https://pro.arcgis.com/en/pro-app/latest/tool-reference/spatial-analyst/train-support-vector-machine-classifier.htm (accessed Nov 20, 2021).

10. Train random trees classifier (spatial analyst) https://pro.arcgis.com/en/pro-app/latest/tool-reference/spatial-analyst/train-random-trees-classifier.htm (accessed Nov 20, 2021).

11. Ha, N. T.; Manley-Harris, M.; Pham, T. D.; Hawes, I. A comparative assessment of Ensemble-based machine learning and maximum likelihood methods for mapping seagrass using sentinel-2 imagery in Tauranga Harbor, New Zealand. Remote Sensing 2020, 12, 355.

12. Otukei, J. R.; Blaschke, T. Land cover change assessment using decision trees, support vector machines and maximum likelihood classification algorithms. International Journal of Applied Earth Observation and Geoinformation 2010, 12.

13. Jamali, A. A fit-for-purpose algorithm for environmental monitoring based on maximum likelihood, support vector machine and Random Forest. The International Archives of the Photogrammetry, Remote Sensing and Spatial Information Sciences 2019, XLII3/W7, 25-32.

14. Rimal, B.; Rijal, S.; Kunwar, R. Comparing support vector machines and maximum likelihood classifiers for mapping of urbanization. Journal of the Indian Society of Remote Sensing 2019, 48, 71-79.

15. Chassagnon, G.; Vakalopoulou, M.; Régent, A.; Zacharaki, E. I.; Aviram, G.; Martin, C.; Marini, R.; Bus, N.; Jerjir, N.; Mekinian, A.; Hua-Huy, T.; Monnier-Cholley, L.; Benmostefa, N.; Mouthon, L.; Dinh-Xuan, A.-T.; Paragios, N.; Revel, M.-P. Deep learning-based approach for automated assessment of interstitial lung disease in systemic sclerosis on CT images. Radiology: Artificial Intelligence 2020, 2.

16. Hinton, G.; Deng, L.; Yu, D.; Dahl, G.; Mohamed, A.-rahman; Jaitly, N.; Senior, A.; Vanhoucke, V.; Nguyen, P.; Sainath, T.; Kingsbury, B. Deep neural networks for acoustic modeling in speech recognition: The shared views of four research groups. IEEE Signal Processing Magazine 2012, 29, 82-97.

17. Litjens, G.; Kooi, T.; Bejnordi, B. E.; Setio, A. A.; Ciompi, F.; Ghafoorian, M.; van der Laak, J. A. W. M.; van Ginneken, B.; Sánchez, C. I. A survey on Deep Learning in medical image analysis. Medical Image Analysis 2017, 42, 60-88.

18. Liu, Y.; Chen, X.; Wang, Z.; Wang, Z. J.; Ward, R. K.; Wang, X. Deep learning for pixel-level image fusion: Recent advances and future prospects. Information Fusion 2018, 42, 158-173.

19. Zhu, X. X.; Tuia, D.; Mou, L.; Xia, G.-S.; Zhang, L.; Xu, F.; Fraundorfer, F. Deep learning in remote sensing: A comprehensive review and list of resources. IEEE Geoscience and Remote Sensing Magazine 2017, 5, 8-36.

20. Ma, L.; Liu, Y.; Zhang, X.; Ye, Y.; Yin, G.; Johnson, B. A. Deep learning in remote sensing applications: A Meta-analysis and Review. ISPRS Journal of Photogrammetry and Remote Sensing 2019, 152, $166-177$.

21. Liu, P.; Choo, K.-K. R.; Wang, L.; Huang, F. SVM or Deep Learning? A comparative study on Remote Sensing Image Classification. Soft Computing 2016, 21, 7053-7065.

22. Li, W.; Fu, H.; Yu, L.; Gong, P.; Feng, D.; Li, C.; Clinton, N. Stacked autoencoder-based deep learning for remote-sensing image classification: A case study of african land-cover mapping. International Journal of Remote Sensing 2016, 37, 5632-5646.

23. Ghorbanzadeh, O.; Blaschke, T.; Gholamnia, K.; Meena, S.; Tiede, D.; Aryal, J. Evaluation of different machine learning methods and deep-learning convolutional neural networks for landslide detection. Remote Sensing 2019, 11, 196.

24. Hartling, S.; Sagan, V.; Sidike, P.; Maimaitijiang, M.; Carron, J. Urban tree species classification using a worldview-2/3 and lidar data fusion approach and Deep Learning. Sensors 2019, 19, 1284.

25. Jozdani, S. E.; Johnson, B. A.; Chen, D. Comparing deep neural networks, ensemble classifiers, and support vector machine algorithms for object-based urban land use/land cover classification. Remote Sensing 2019, 11, 1713.

26. Nijhawan, R.; Sharma, H.; Sahni, H.; Batra, A. A deep learning hybrid CNN framework approach for vegetation cover mapping using deep features. 2017 13th International Conference on Signal-Image Technology E Internet-Based Systems (SITIS) 2017.

27. Wan, J.; Ma, Y. Multi-scale spectral-spatial remote sensing classification of coral reef habitats using CNN-SVM. Journal of Coastal Research 2020, 102.

28. Ronneberger, O.; Fischer, P.; Brox, T. U-Net: Convolutional Networks for Biomedical Image Segmentation. Lecture Notes in Computer Science 2015, 234-241.

29. McGlinchy, J.; Johnson, B.; Muller, B.; Joseph, M.; Diaz, J. Application of unet fully convolutional neural network to impervious surface segmentation in urban environment from high resolution satellite imagery. IGARSS 2019 - 2019 IEEE International Geoscience and Remote Sensing Symposium 2019.

30. Li, H.; Wang, C.; Cui, Y.; Hodgson, M. Mapping salt marsh along coastal South Carolina using U-Net. ISPRS Journal of Photogrammetry and Remote Sensing 2021, 179, 121-132.

31. Dang, K. B.; Nguyen, M. H.; Nguyen, D. A.; Phan, T. T.; Giang, T. L.; Pham, H. H.; Nguyen, T. N.; Tran, T. T.; Bui, D. T. Coastal wetland classification with deep U-Net Convolutional Networks and sentinel-2 imagery: A case study at the Tien Yen Estuary of Vietnam. Remote Sensing 2020, 12, 3270.

32. James, G.; Witten, D.; Hastie, T.; Tibshirani, R. An introduction to statistical learning: With applications in R; Springer: New York, NY, 2013.

33. Mountrakis, G.; Im, J.; Ogole, C. Support Vector Machines in remote sensing: A Review. ISPRS Journal of Photogrammetry and Remote Sensing 2011, 66, 247-259.

34. Bahari, N. I.; Ahmad, A.; Aboobaider, B. M. Application of support vector machine for classification of Multispectral Data. IOP Conference Series: Earth and Environmental Science 2014, 20, 012038. 
35. Pal, M. Random forest classifier for remote sensing classification. International Journal of Remote Sensing 2005, 26, $217-222$.

36. Kennish, M. J. Environmental threats and environmental future of Estuaries. Environmental Conservation 2002, $29,78-107$.

37. Sanger, D.; Blair, A.; DiDonato, G.; Washburn, T.; Jones, S.; Riekerk, G.; Wirth, E.; Stewart, J.; White, D.; Vandiver, L.; Holland, A. F. Impacts of coastal development on the ecology of Tidal Creek ecosystems of the US southeast including consequences to humans. Estuaries and Coasts 2013, 38, 49-66.

38. Hong, S.-K.; Koh, C.-H.; Harris, R. R.; Kim, J.-E.; Lee, J.-S.; Ihm, B.-S. Land use in Korean tidal wetlands: Impacts and management strategies. Environmental Management 2008, 45, 1014-1026.

39. Alber, M.; Swenson, E. M.; Adamowicz, S. C.; Mendelssohn, I. A. Salt marsh dieback: an overview of recent events in the US. Estuarine, Coastal and Shelf Science 2008, 80(1), 1-11.

40. U.S. Census Bureau QuickFacts: Beaufort County, South Carolina. Available online: https://www.census.gov/quickfacts/beaufortcountysouthcarolina (accessed on 20 Nov 2021).

41. Port Royal Sound. Available online: https://www.lowcountryinstitute.org/prs (accessed on 20 Nov 2021).

42. Purcell, A. D.; Khanal, P.; Straka, T.; Willis, D. B. Valuing ecosystem services of coastal marshes and wetlands. Land-Grant Press by Clemson Extension 2020.

43. Willis, D.B.; Straka T.J. The economic contribution of natural resources to South Carolina's economy. Bulletin FW 13. Clemson, SC: Clemson University Experiment Station. 2016.

44. Sea Level Rise Adaptation Report Beaufort County, South Carolina. SC Sea Grant Consortium Product \#SCSGC-T-15-02. 2015.

45. Beaufort County Comprehensive Plan Chapter 5. Available online: https://www.beaufortcountysc.gov/council/comprehensiveplan/documents/2010-comprehensive-plan-documents/chapter-5-natural-resources.pdf (accessed on 20 Nov 2021).

46. Davis, D. National Agriculture Imagery Program (NAIP) Information Sheet. https://www.fsa.usda.gov/Assets/USDA-FSAPublic/usdafiles/APFO/support-documents/pdfs/naip_infosheet_2016.pdf, 2017.

47. Download Seamless Wetlands Data by State. Available online: https://www.fws.gov/wetlands/data/State-Downloads.html (accessed on (20 Nov 2021)

48. Microsoft Microsoft/USBUILDINGFOOTPRINTS: Computer generated building footprints for the United States https://github.com/Microsoft/USBuildingFootprints (accessed Nov 20, 2021).

49. Huang, X.; Wang, C.; Li, Z.; Ning, H. A $100 \mathrm{~m}$ population grid in the CONUS by disaggregating census data with open-source Microsoft Building Footprints. Big Earth Data 2020, 5, 112-133.

50. Anderson, J. R.; Hardy, E. E.; Roach, J. T.; Witmer, R. E. A land use and land cover classification system for use with Remote Sensor Data. Professional Paper 1976.

51. Blaschke, T.; Hay, G. J.; Kelly, M.; Lang, S.; Hofmann, P.; Addink, E.; Queiroz Feitosa, R.; van der Meer, F.; van der Werff, H.; van Coillie, F.; Tiede, D. Geographic object-based image analysis - towards a new paradigm. ISPRS Journal of Photogrammetry and Remote Sensing 2014, 87, 180-191.

52. Ouyang, Z.-T.; Zhang, M.-Q.; Xie, X.; Shen, Q.; Guo, H.-Q.; Zhao, B. A comparison of pixel-based and object-oriented approaches to VHR imagery for mapping saltmarsh plants. Ecological Informatics 2011, 6, 136-146.

53. Li, X.; Shao, G. Object-based land-cover mapping with high resolution aerial photography at a county scale in Midwestern USA. Remote Sensing 2014, 6, 11372-11390.

54. Whiteside, T. G.; Boggs, G. S.; Maier, S. W. Comparing object-based and pixel-based classifications for mapping savannas. International Journal of Applied Earth Observation and Geoinformation 2011, 13, 884-893.

55. Congalton, R.; Oderwald, R. G.; Mead, R. (1983). Assessing Landsat classification accuracy using discrete multivariate statistical techniques. Photogrammetric Engineering and Remote Sensing 1983, 1671-1678.

56. Jensen, J. R. Introductory digital image processing: A remote sensing perspective, 4th ed.; Pearson Education: Glenview, IL, USA, 2016.

57. Wedge, M.; Anderson, C. J.; DeVries, D. Evaluating the effects of urban land use on the condition of resident salt marsh fish. Estuaries and Coasts 2015, 38, 2355-2365.

58. Zhang, W.; Tang, P.; Zhao, L. Fast and accurate land-cover classification on medium-resolution remote-sensing images using segmentation models. International Journal of Remote Sensing 2021, 42, 3277-3301.

59. DeLancey, E. R.; Simms, J. F.; Mahdianpari, M.; Brisco, B.; Mahoney, C.; Kariyeva, J. Comparing deep learning and shallow learning for large-scale wetland classification in Alberta, Canada. Remote Sensing 2019, 12, 2.

60. Liu, T.; Abd-Elrahman, A.; Morton, J.; Wilhelm, V. L. Comparing fully convolutional networks, random forest, support vector machine, and patch-based deep convolutional neural networks for object-based wetland mapping using images from small unmanned aircraft system. GIScience E Remote Sensing 2018, 55, 243-264.

61. Mahdianpari, M.; Rezaee, M.; Zhang, Y.; Salehi, B. Wetland classification using Deep Convolutional Neural Network. IGARSS 2018 - 2018 IEEE International Geoscience and Remote Sensing Symposium 2018.

62. Du, L.; McCarty, G. W.; Zhang, X.; Lang, M. W.; Vanderhoof, M. K.; Li, X.; Huang, C.; Lee, S.; Zou, Z. Mapping forested wetland inundation in the Delmarva Peninsula, USA using deep convolutional Neural Networks. Remote Sensing 2020, $12,644$.

63. Gallant, A. The challenges of remote monitoring of wetlands. Remote Sensing 2015, 7, 10938-10950.

64. Tiner, R.W. Introduction to Wetland Mapping and Its Challenges. In Remote Sensing of Wetlands: Applications and Advances 1st ed.; R.W. Tiner, M.W. Lang \& V.V. Klemas (Eds.); CRC Press, 2015; pp. 43-65.

65. O'Donnell, J.; Schalles, J. Examination of abiotic drivers and their influence on Spartina alterniflora biomass over a twenty-eight year period using Landsat 5 TM satellite imagery of the central Georgia coast. Remote Sensing 2016, 8, 477.

66. Doughty, C.; Cavanaugh, K. Mapping coastal wetland biomass from high resolution unmanned aerial vehicle (UAV) imagery. Remote Sensing 2019, 11, 540. 
67. Ai, J.; Gao, W.; Gao, Z.; Shi, R.; Zhang, C. Phenology-based Spartina alterniflora mapping in coastal wetland of the Yangtze Estuary using time series of Gaofen Satellite No. 1 wide field of view imagery. Journal of Applied Remote Sensing 2017, 11, 026020.

68. Lang, M.W., Purkis, S., Klemas, V.V., \& Tiner R.W. Promising Developments and Future Challenges for Remote Sensing of Wetlands. In Remote Sensing of Wetlands: Applications and Advances 1st ed.; R.W. Tiner, M.W. Lang \& V.V. Klemas (Eds.); CRC Press, 2015; pp. 533-544.

69. Dehouck, A.; Lafon, V.; Baghdadi, N.; Marieu, V. Use of optical and radar data in synergy for mapping intertidal flats and coastal salt-marshes (arcachon lagoon, France). 2012 IEEE International Geoscience and Remote Sensing Symposium 2012.

70. Ramsey III, E., \& Rangoonwala, A. Radar and Optical Image Fusion and Mapping of Wetland Resources. In Remote Sensing of Wetlands: Applications and Advances 1st ed.; R.W. Tiner, M.W. Lang \& V.V. Klemas (Eds.); CRC Press, 2015; pp. 155-174.

71. Difebo, A., Richardson, M., \& Price, J. Fusion of Multispectral Imagery and LiDAR Digital Terrain Derivatives for Ecosystem Mapping and Morphological Characterization of a Northern Peatland Complex. In Remote Sensing of Wetlands: Applications and Advances 1st ed.; R.W. Tiner, M.W. Lang \& V.V. Klemas (Eds.); CRC Press, 2015; pp. 399-412.

72. Schill, S. R.; Jensen, J. R. Predicting the impact of coastal development on water quality using remote sensing and gis-assisted hydrologic modeling techniques. Geocarto International 2000, 15, 7-16. 